\title{
Comparing the long-term efficacy of standard and combined minimally invasive procedures for unresectable HCC: a mixed treatment comparison
}

\author{
Jianghai Zhao', ${ }^{1,}$, Hui Zhang ${ }^{1, *}$, Lunshou Wei ${ }^{1}$, Shuping Xie ${ }^{1}$, Zhimin Suo ${ }^{1}$ \\ ${ }^{1}$ Department of Gastroenterology, Huaihe Hospital of Henan University, Kaifeng, Henan, 475000, China \\ *These authors have contributed equally to this work \\ Correspondence to: Zhimin Suo, email: wenyuhuwy@126.com \\ Keywords: unresectable hepatocellular carcinoma, transarterial chemoembolization, long-term efficacy, network meta-analysis \\ Received: May 23, 2016 \\ Accepted: October 12, 2016 \\ Published: November 07, 2016
}

\section{ABSTRACT}

A small proportion of hepatocellular carcinoma (HCC) patients are suitable for surgical resections and various minimally invasive procedures have been introduced as alternatives to surgical resections. However, the relative efficacy of minimally invasive procedures remains to be studied in the current literature. Several popular minimally invasive procedures (monotherapy or combined therapies) were selected for comparison and their relative long-term efficacy were determined by using the statistics of hazard ratio (HR) which evaluates the survival status of HCC patients in one, two, three and four years, respectively. Evidence were obtained from the current literature and synthesized by using the approach of conventional pairwise metaanalysis and network meta-analysis (NMA). Moreover, selected minimally invasive procedures were ranked according to their surface under the cumulative ranking curve (SUCRA) which was produced by NMA in conjunction with the Markov Chain Monte Carlo (MCMC) sampling method. HCC patients treated by combined minimally invasive procedures, particularly transcatheter arterial chemoembolization (TACE) + high intensity focused ultrasound (HIFU), TACE + radiofrequency ablation (RFA), TACE + radiotherapy (RT) and TACE + Sorafenib (SOR) exhibited a significant decrease in the HR compared to those with standard TACE $(H R<1)$. The combined minimally invasive procedure of TACE + HIFU appears to be the most preferable therapy. PEI seems to be less favorable than other minimally invasive procedures. Combined minimally invasive procedures may be more preferable than standard minimally invasive procedures. Percutaneous ethanol injection (PEI) may not provide adequate efficacy compared to other minimally invasive procedures for unresectable HCC patients.

\section{INTRODUCTION}

Hepatocellular carcinoma (HCC) is one of the five most common cancers worldwide and it is a prototype of inflammation-associated malignancies [1]. Although surgical operations have been prioritized for HCC patients, complete resection is not appropriate for patients who are diagnosed in advanced stages [2]. On the other hand, transarterial chemoembolization (TACE) is a standard minimally invasive procedure developed for HCC patients who are not eligible for complete resection [3]. TACE involves the injection of a chemotherapeutic agent, which induces selective vascular embolization and blocks the arteries, hence triggering tumor infarction and necrosis
$[4,5]$. The partial response rate for unresectable HCC patients with TACE is approximately $62 \%$ and TACE is able to prominently suppress tumor vascular invasion and progression [4]. However, TACE has its own limitations [6], for instance, TACE may further affect liver functions and damage the hepatic arterial system. As a result of this, TACE is not appropriate for patients with poor liver functions, particularly those with cirrhosis [3, 7].

Recently, new approaches have been introduced as either standard minimally invasive procedures or adjuvant therapies in order to improve the survival status of HCC patients: yttrium-90 radioembolization (TARE-90Y), radiotherapy (RT), percutaneous acetic acid injection (PAI), external-beam radiation therapy (EBRT), drug-eluting 
beads-transcatheter arterial chemoembolization (DEBTACE), percutaneous ethanol injection (PEI) and sorafenib (SOR) [8-13]. Studies in the current literature suggest that standard minimally invasive procedures in combination with adjuvant therapies may be more efficacious than monotherapy. For instance, Li et al. concluded in their study that patients with TACE + high-intensity focused ultrasound (HIFU) exhibited significantly higher survival rate compared to those with TACE [14]. Moreover, evidence also indicated that combined strategy of TACE + PEI/RFA is more efficacious than the monotherapy of TACE with respect to long term survival rates [15]. Besides that, the combination therapy of TACE with radiofrequency ablation (RFA) may have several theoretical advantages compared to RFA alone, and patients with TACE-RFA exhibited a higher overall survival rate compared to those with monotherapy [6]. Furthermore, combining TACE with RT may trigger synergistic effects and enhance the efficacy of monotherapy [16]. However, the lack of a systematic review inspired us to compare standard minimally invasive procedures with combined therapies in order to benefit patients with unresectable HCC.

This study extended the scope of conventional metaanalysis by incorporating indirect evidence that can be obtained from clinical trials. It is anticipated that using this approach enabled us to determine the relative efficacy of standard or combined minimally invasive procedures without concerning about the ethical issues resulted from designing new randomized clinical trials.

\section{RESULTS}

\section{Baseline characteristics of the included studies}

In total 42 articles (Table 1) were selected and included in the study after screening out irrelevant papers [17-58]. Among the total 5,666 subjects, 2,392 (42.22\%) individuals underwent TACE treatment and 891 (15.73\%) individuals received RFA treatment. Furthermore, 438 (7.73\%), $432(7.62 \%), 379(6.69 \%), 325(5.74 \%), 310$ $(5.47 \%), 174(3.07 \%), 131(2.31 \%), 68(1.20 \%), 63$ $(1.11 \%), 54(0.95 \%)$ and $9(0.16 \%)$ patients underwent TACE+RFA, PEI, TACE + SOR, DEB-TACE, TARE-90Y, TACE + PEI, TACE + RT, TACE + HIFU, PAI, TACE + EBRT and RT respectively. Aside from four trials that were three-arm trials, 38 were two-arm trials and a total of 16 comparisons were created among the 42 studies. In term of OS-1 (Figure 1A), there are 40 studies providing data for 16 comparisons. For OS-2 and OS-3 (Figure 1A), data from 41 and 36 trials are provided, respectively. For OS-4 (Figure 1B), data were provided by 23 studies.

\section{Pairwise meta-analysis}

We completed the pairwise meta-analysis for the 16 comparisons and the weighted HRs for each comparison was calculated. The results of the pair-wise comparisons is shown in Figure 1 which illustrates the comparison of OS-1, OS-2, OS-3 (Figure 1A) and OS-4 (Figure 1B). For OS-1 (Table 2), direct comparisons suggest that combined TACE therapies, as well as RFA and RT, was more efficacious than TACE monotherapy (all HR $<1$ and $95 \% \mathrm{CI}$ of HR exclude 1). PEI appeared to be less efficacious than its counterpart TACE + PEI $(\mathrm{HR}=2.04$, $95 \%$ CI, 1.69 - 2.56). Similarly, the efficacy of RFA was worse than that of TACE + RFA $(\mathrm{HR}=2.13,95 \% \mathrm{CI}, 1.92$ - 2.44) whereas RFA exhibits better efficacy compared to PEI $(\mathrm{HR}=0.30,95 \% \mathrm{CI}, 0.20-0.41)$.

For OS-2 (Table 3), the majority of the selected therapies exhibited a greater efficacy compared to TACE monotherapy (HR $<1$ and $95 \%$ CI of HR exclude 1 ). Besides that, PEI monotherapy appeared to be less efficacious than the combined approach of TACE + PEI $(\mathrm{HR}=2.02,95 \% \mathrm{CI}, 1.77-2.35)$. Likewise, patients with RFA exhibited an increased HR compared to those with TACE + RFA $(\mathrm{HR}=1.90,95 \%$ CI, $1.66-2.22)$. Finally, RT monotherapy was far less effective than the combined approach of TACE + RT $(\mathrm{HR}=5.56,95 \% \mathrm{CI}, 4.44-7.41)$.

Direct comparisons in Table 4 indicated that TACE + EBRT, TACE + HIFU, TACE + PEI, TACE + RFA, TACE + RT, TACE + SOR, TARE-90Y, DEB-TACE, RFA and RT were more effective than TACE monotherapy with respect to OS-3 (HR $<1$ and $95 \%$ CI of HR exclude 1 ). In addition, patients treated with RFA had higher OS3 compared to those treated with PEI ( $\mathrm{HR}=0.48,95 \% \mathrm{CI}$, $0.41-0.55)$. On the other hand, RFA was less effective than TACE + RFA $(\mathrm{HR}=1.76,95 \% \mathrm{CI}, 1.56-2.00)$ and RT was less effective than TACE + RT $(\mathrm{HR}=5.56,95 \%$ CI, 4.44 - 7.41). The comparison results displayed in Table 5 were very similar to the results mentioned above.

\section{Network meta-analysis}

As suggested by the lower off-diagonal area in Tables 2-5, a large number of comparisons were generated by the network meta-analysis. As for OS-1, combined therapies including TACE + HIFU, TACE + RFA, TACE $+\mathrm{RT}$, TACE + SOR and DEB-TACE appeared to be more effective than TACE monotherapy (HR $<1,95 \% \mathrm{CrI}$ excludes 1). By contrast, TACE + PEI, TARE - 90Y, DEB - TACE, PEI, RFA and RT were less effective than TACE + HIFU $(\mathrm{HR}>1,95 \% \mathrm{CrI}$ excludes 1$)$.

Likewise, results from network meta-analysis with respect to OS-2 were displayed in Table 3. Patients treated with combined therapies including TACE + HIFU, TACE + RFA, TACE + RT, TACE + SOR and DEB-TACE were associated with an increased OS-2 in comparison to those treated with TACE monotherapy ( $\mathrm{HR}<1,95 \% \mathrm{CrI}$ excludes 1). Apart from that, TACE + PEI, TACE + SOR, TARE - 90Y, DEB - TACE, PEI, RFA and RT were less effective than TACE+HIFU (HR $>1,95 \%$ CrI excludes 1). Furthermore, patients treated with TACE+RFA and 
Table 1: the main characteristics of included studies

\begin{tabular}{|c|c|c|c|c|c|c|c|}
\hline Study & Region & Year & Treatment 1 & Treatment 2 & Size1 & Size2 & Outcomes \\
\hline Peng (2013) & China & 2013 & TACE+RFA & RFA & 94 & 95 & (1)(2)(3)(4) \\
\hline $\begin{array}{l}\text { Adnan Muhammad } \\
\text { (2013) }\end{array}$ & USA & 2013 & $\mathrm{TACE}+\mathrm{SOR}$ & TACE & 13 & 30 & (1)(2)(3)(4) \\
\hline Wei Bai (2013) & China & 2013 & TACE+SOR & TACE & 82 & 164 & (1)(2) \\
\hline Nicolini (2013) & Italy & 2013 & DEB-TACE & TACE & 22 & 16 & (1)(2)(3)(4) \\
\hline Moreno-Luna (2013) & USA & 2013 & TARE-90Y & TACE & 61 & 55 & (1)(2)(3)(4) \\
\hline Xu-Dong Qu (2012) & China & 2012 & TACE+SOR & TACE & 45 & 45 & (1)(2)(3)(4) \\
\hline Song (2012) & Korea & 2012 & DEB-TACE & TACE & 60 & 69 & (1)(2)(3) \\
\hline Recchia (2012) & Italy & 2012 & DEB-TACE & TACE & 35 & 70 & (1)(2)(3) \\
\hline Peng (2011) & China & 2011 & $\mathrm{TACE}+\mathrm{RFA}$ & RFA & 69 & 70 & (1)(2)(3)(4) \\
\hline $\begin{array}{l}\text { Masatoshi Kudo } \\
\text { (2011) }\end{array}$ & $\begin{array}{c}\text { Japan+South } \\
\text { Korea }\end{array}$ & 2011 & TACE+SOR & TACE & 229 & 229 & (1)(2)(3) \\
\hline Song (2011) & Korea & 2011 & DEB-TACE & TACE & 20 & 20 & (1)(2)(3) \\
\hline Salem (2011) & USA & 2011 & TARE-90Y & TACE & 123 & 122 & (1)(2)(3)(4) \\
\hline Wiggerman (2011) & Germany & 2011 & DEB-TACE & TACE & 22 & 22 & (1)(2)(3) \\
\hline $\operatorname{Sacco}(2011)$ & Italy & 2011 & DEB-TACE & TACE & 33 & 34 & (1)(2)(3) \\
\hline Malagari (2011) & Greece & 2011 & DEB-TACE & TACE & 41 & 43 & (1) \\
\hline $\operatorname{Kim}(2010)$ & Korea & 2010 & $\mathrm{TACE}+\mathrm{RFA}$ & RFA & 83 & 231 & (1)(2)(3)(4) \\
\hline Morimoto (2010) & Japan & 2010 & $\mathrm{TACE}+\mathrm{RFA}$ & RFA & 19 & 18 & (1)(2)(3)(4) \\
\hline $\mathrm{Li}(2010)$ & China & 2010 & $\mathrm{TACE}+\mathrm{HIFU}$ & TACE & 44 & 45 & (1)(2)(3)(4) \\
\hline $\operatorname{Tan}(2010)$ & China & 2010 & TACE+SOR & TACE & 10 & 10 & (1)(2) \\
\hline Kooby (2010) & USA & 2010 & TARE-90Y & TACE & 27 & 44 & (1)(2)(3)(4) \\
\hline Carr (2010) & USA & 2010 & TARE-90Y & TACE & 99 & 691 & (1)(2)(3) \\
\hline Ferrer Puchol (2010) & Spain & 2010 & DEB-TACE & TACE & 47 & 25 & (1)(2)(3)(4) \\
\hline Dhanasekaran (2010) & USA & 2010 & DEB-TACE & TACE & 45 & 26 & (1)(2) \\
\hline Shibata (2009) & Japan & 2009 & $\mathrm{TACE}+\mathrm{RFA}$ & TACE & 46 & 43 & (2)(3)(4) \\
\hline Yang-a (2009) & China & 2009 & $\mathrm{TACE}+\mathrm{RFA}$ & RFA & 31 & 37 & (1)(2)(3)(4) \\
\hline Yang-c (2009) & China & 2009 & RFA & TACE & 37 & 35 & (1)(2)(3)(4) \\
\hline Yang-b (2009) & China & 2009 & $\mathrm{TACE}+\mathrm{RFA}$ & TACE & 31 & 35 & (1)(2)(3)(4) \\
\hline Cheng-b (2008) & China & 2008 & $\mathrm{TACE}+\mathrm{RFA}$ & TACE & 96 & 95 & (1)(2)(3)(4) \\
\hline Cheng-a (2008) & China & 2008 & $\mathrm{TACE}+\mathrm{RFA}$ & RFA & 96 & 100 & (1)(2)(3)(4) \\
\hline Cheng-c (2008) & China & 2008 & RFA & TACE & 100 & 95 & (1)(2)(3)(4) \\
\hline Brunello (2008) & Italy & 2008 & RFA & PEI & 70 & 69 & (1)(2)(3)(4) \\
\hline Wu (2005) & China & 2005 & TACE+HIFU & TACE & 24 & 26 & (1)(2) \\
\hline Becker (2005) & Germany & 2005 & TACE+PEI & TACE & 27 & 25 & (1)(2)(3) \\
\hline Shiina (2005) & Japan & 2005 & RFA & PEI & 118 & 114 & (1)(2)(3)(4) \\
\hline $\operatorname{Lin}(2005)$ & Taiwan & 2005 & PAI & PEI & 63 & 62 & (1)(2)(3) \\
\hline
\end{tabular}




\begin{tabular}{|c|c|c|c|c|c|c|c|}
\hline Study & Region & Year & Treatment 1 & Treatment 2 & Size1 & Size2 & Outcomes \\
\hline Shim (2005) & Korea & 2005 & $\mathrm{TACE}+\mathrm{RT}$ & TACE & 38 & 35 & (1)(2)(3) \\
\hline Lin-a (2004) & Taiwan & 2004 & RFA & PEI & 50 & 46 & (1)(2)(3) \\
\hline Lin-b (2004) & Taiwan & 2004 & RFA & PEI & 50 & 50 & (1)(2)(3) \\
\hline Zeng (2004) & China & 2004 & TACE+EBRT & TACE & 54 & 149 & (1)(2)(3)(4) \\
\hline Lencioni (2003) & Italy & 2003 & RFA & PEI & 52 & 50 & (1)(2)(3) \\
\hline Guo (2003) & China & 2003 & $\mathrm{TACE}+\mathrm{RT}$ & TACE & 76 & 89 & (1)(2)(3) \\
\hline Kamada (2002) & Japan & 2002 & TACE+PEI & TACE & 32 & 37 & (1)(2)(3) \\
\hline Koda (2001) & Japan & 2001 & TACE+PEI & PEI & 26 & 26 & (2)(3)(4) \\
\hline $\begin{array}{l}\text { Chia-Hsien Cheng-b } \\
\text { (2001) }\end{array}$ & China & 2001 & $\mathrm{TACE}+\mathrm{RT}$ & RT & 17 & 9 & (1)(2)(3) \\
\hline $\begin{array}{l}\text { Chia-Hsien Cheng-c } \\
\text { (2001) }\end{array}$ & China & 2001 & RT & TACE & 9 & 16 & (1)(2)(3)(4) \\
\hline $\begin{array}{l}\text { Chia-Hsien Cheng-a } \\
\text { (2001) }\end{array}$ & China & 2001 & TACE+RT & TACE & 17 & 16 & (1)(2)(3)(4) \\
\hline Allgaier-b (1998) & Germany & 1998 & TACE+PEI & TACE & 39 & 33 & (1)(2) \\
\hline Allgaier-a (1998) & Germany & 1998 & TACE+PEI & PEI & 39 & 15 & (1)(2) \\
\hline Allgaier-c (1998) & Germany & 1998 & PEI & TACE & 15 & 33 & (1)(2) \\
\hline Bartolozzi (1995) & Italy & 1995 & TACE+PEI & TACE & 26 & 27 & (1)(2)(3) \\
\hline Kato (1994) & Japan & 1994 & TACE+PEI & TACE & 24 & 22 & (1)(2)(3) \\
\hline
\end{tabular}

1. TACE: transcatheter arterial chemoembolization; EBRT: external-beam radiation therapy; HIFU: high intensity focused ultrasound; PEI: percutaneous ethanol injection; RFA: radiofrequency ablation; RT: radiotherapy; SOR: sorafenib; TARE90Y: yttrium-90 radioembolization; DEB-TACE: drug-eluting beads-transcatheter arterial chemoembolization; PAI: percutaneous acetic acid injection.

2. Outcomes: (1)-overall survival of 1 year; (2)-overall survival of 2 years; (3)-overall survival of 3 years; (4)-overall survival of 4 years

(A) OS-1 year, OS-2 year, OS-3 year

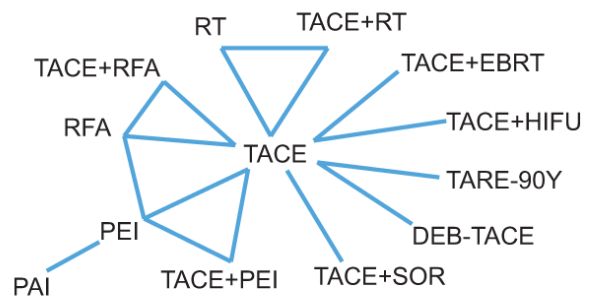

(B) OS-4 year

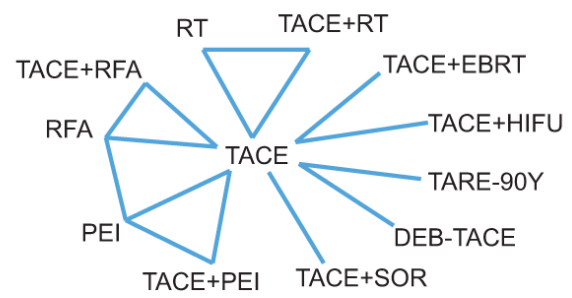

Figure 1: Network design of 13 therapies in the network meta-analysis A. OS-1, OS-2, OS-3; B. OS-4. 
Table 2: Comparing the relative efficacy of therapies with respect to one-year survival status using pairwise and network meta-analysis

\begin{tabular}{|c|c|c|c|c|c|c|c|c|c|c|c|c|c|}
\hline & TACE & $\begin{array}{c}\text { TACE } \\
+ \text { EBRT }\end{array}$ & $\begin{array}{c}\text { TACE } \\
+ \text { HIFU }\end{array}$ & $\begin{array}{l}\text { TACE } \\
+ \text { PEI }\end{array}$ & $\begin{array}{l}\text { TACE } \\
+ \text { RFA }\end{array}$ & $\begin{array}{l}\text { TACE } \\
+ \text { RT }\end{array}$ & $\begin{array}{l}\text { TACE } \\
+ \text { SOR }\end{array}$ & $\begin{array}{l}\text { TARE } \\
-90 Y\end{array}$ & DEB-TACE & PAI & PEI & RFA & RT \\
\hline TACE & & $\begin{array}{c}0.73(0.56, \\
0.90)\end{array}$ & $\begin{array}{c}0.24(0.21 \\
0.28)\end{array}$ & $\begin{array}{c}0.48(0.41, \\
0.54)\end{array}$ & $\begin{array}{c}0.52(0.39 \\
0.66)\end{array}$ & $\begin{array}{c}0.52(0.45 \\
0.60)\end{array}$ & $\begin{array}{c}0.47(0.45, \\
0.49)\end{array}$ & $\begin{array}{c}0.88(0.80, \\
0.97)\end{array}$ & $\begin{array}{c}0.51(0.46 \\
0.57)\end{array}$ & - & $\begin{array}{c}0.91(0.78, \\
1.04)\end{array}$ & $\begin{array}{c}0.84(0.67 \\
1.00)\end{array}$ & $\begin{array}{c}0.42(0.32, \\
0.52)\end{array}$ \\
\hline TACE+EBRT & $\begin{array}{c}0.73(0.30 \\
1.75)\end{array}$ & & - & - & - & - & - & - & - & - & - & - & - \\
\hline TACE+HIFU & $\begin{array}{c}0.31(0.17 \\
0.57)\end{array}$ & $\begin{array}{c}0.42(0.14, \\
1.22)\end{array}$ & & - & - & - & - & - & - & - & - & - & - \\
\hline TACE+PEI & $\begin{array}{c}0.72(0.47 \\
1.09)\end{array}$ & $\begin{array}{c}0.99(0.37, \\
2.60)\end{array}$ & $\begin{array}{c}2.35(1.12 \\
4.95)\end{array}$ & & - & - & - & - & - & - & $\begin{array}{c}2.04(1.69 \\
2.56)\end{array}$ & & \\
\hline TACE+RFA & $\begin{array}{c}0.45(0.28 \\
0.72)\end{array}$ & $\begin{array}{c}0.62(0.23, \\
1.67)\end{array}$ & $\begin{array}{c}1.48(0.68 \\
3.21)\end{array}$ & $\begin{array}{c}0.63(0.34, \\
1.15)\end{array}$ & & - & - & - & - & - & - & $\begin{array}{c}2.13(1.92 \\
2.44)\end{array}$ & \\
\hline TACE+RT & $\begin{array}{c}0.41(0.25 \\
0.66)\end{array}$ & $\begin{array}{c}0.56(0.21, \\
1.51)\end{array}$ & $\begin{array}{c}1.33(0.61 \\
2.90)\end{array}$ & $\begin{array}{c}0.56(0.30 \\
1.07)\end{array}$ & $\begin{array}{c}0.90(0.46 \\
1.76)\end{array}$ & & - & - & - & - & - & - & $\begin{array}{c}6.25(5.00, \\
8.33)\end{array}$ \\
\hline TACE+SOR & $\begin{array}{c}0.55(0.37 \\
0.83)\end{array}$ & $\begin{array}{c}0.76(0.29 \\
1.99)\end{array}$ & $\begin{array}{c}1.80(0.86 \\
3.78)\end{array}$ & $\begin{array}{c}0.77(0.43, \\
1.38)\end{array}$ & $\begin{array}{c}1.22(0.65 \\
2.28)\end{array}$ & $\begin{array}{c}1.36(0.72 \\
2.56)\end{array}$ & & - & - & - & - & - & - \\
\hline TARE-90Y & $\begin{array}{c}0.88(0.57 \\
1.35)\end{array}$ & $\begin{array}{c}1.20(0.45, \\
3.19)\end{array}$ & $\begin{array}{c}2.86(1.35 \\
6.07)\end{array}$ & $\begin{array}{c}1.22(0.67, \\
2.22)\end{array}$ & $\begin{array}{c}1.93(1.02, \\
3.67)\end{array}$ & $\begin{array}{l}2.15(1.13 \\
\quad 4.12)\end{array}$ & $\begin{array}{c}1.59(0.87, \\
2.89)\end{array}$ & & - & - & - & - & - \\
\hline DEB-TACE & $\begin{array}{c}0.73(0.53 \\
1.00)\end{array}$ & $\begin{array}{c}0.99(0.39, \\
2.53)\end{array}$ & $\begin{array}{c}2.37(1.18 \\
4.75)\end{array}$ & $\begin{array}{c}1.01(0.59, \\
1.71)\end{array}$ & $\begin{array}{c}1.60(0.91, \\
2.84)\end{array}$ & $\begin{array}{c}1.79(1.00 \\
3.19)\end{array}$ & $\begin{array}{c}1.32(0.78 \\
2.22)\end{array}$ & $\begin{array}{c}0.83(0.48, \\
1.42)\end{array}$ & & - & - & - & - \\
\hline PAI & $\begin{array}{c}1.00(0.34 \\
2.91)\end{array}$ & $\begin{array}{c}1.37(0.34, \\
5.44)\end{array}$ & $\begin{array}{c}3.27(0.95 \\
11.19)\end{array}$ & $\begin{array}{c}1.39(0.46 \\
4.19)\end{array}$ & $\begin{array}{c}2.21(0.74 \\
6.63)\end{array}$ & $\begin{array}{c}2.46(0.76 \\
7.93)\end{array}$ & $\begin{array}{c}1.81(0.58 \\
5.69)\end{array}$ & $\begin{array}{c}1.14(0.36, \\
3.61)\end{array}$ & $\begin{array}{c}1.38(0.45 \\
4.20)\end{array}$ & & $\begin{array}{c}1.33(0.90 \\
2.56)\end{array}$ & - & - \\
\hline PEI & $\begin{array}{c}1.33(0.84 \\
2.13)\end{array}$ & $\begin{array}{c}1.83(0.68, \\
4.92)\end{array}$ & $\begin{array}{l}4.35(2.01 \\
9.42)\end{array}$ & $\begin{array}{c}1.85(1.07, \\
3.19)\end{array}$ & $\begin{array}{c}2.95(1.72, \\
5.04)\end{array}$ & $\begin{array}{l}3.28(1.68 \\
\quad 6.41)\end{array}$ & $\begin{array}{c}2.42(1.30 \\
4.50)\end{array}$ & $\begin{array}{c}1.52(0.80, \\
2.88)\end{array}$ & $\begin{array}{c}1.84(1.04 \\
3.24)\end{array}$ & $\begin{array}{c}1.33(0.51, \\
3.48)\end{array}$ & & $\begin{array}{c}0.30(0.20 \\
0.41)\end{array}$ & - \\
\hline RFA & $\begin{array}{c}0.87(0.57 \\
1.32)\end{array}$ & $\begin{array}{c}1.19(0.45, \\
3.13)\end{array}$ & $\begin{array}{c}2.83(1.34 \\
5.95)\end{array}$ & $\begin{array}{c}1.20(0.70 \\
2.08)\end{array}$ & $\begin{array}{c}1.91(1.27, \\
2.88)\end{array}$ & $\begin{array}{c}2.13(1.13 \\
4.03)\end{array}$ & $\begin{array}{c}1.57(0.87, \\
2.82)\end{array}$ & $\begin{array}{c}0.99(0.54, \\
1.81)\end{array}$ & $\begin{array}{c}1.19(0.70 \\
2.02)\end{array}$ & $\begin{array}{c}0.87(0.31, \\
2.45)\end{array}$ & $\begin{array}{c}0.65(0.44 \\
0.97)\end{array}$ & & - \\
\hline RT & $\begin{array}{c}1.03(0.53 \\
2.01)\end{array}$ & $\begin{array}{c}1.41(0.47, \\
4.24)\end{array}$ & $\begin{array}{c}3.37(1.36 \\
8.34)\end{array}$ & $\begin{array}{c}1.43(0.65 \\
3.15)\end{array}$ & $\begin{array}{c}2.28(1.01 \\
5.15)\end{array}$ & $\begin{array}{c}2.54(1.30 \\
\quad 4.94)\end{array}$ & $\begin{array}{c}1.87(0.85 \\
4.09)\end{array}$ & $\begin{array}{c}1.18(0.53, \\
2.61)\end{array}$ & $\begin{array}{c}1.42(0.68 \\
2.98)\end{array}$ & $\begin{array}{c}1.03(0.29, \\
3.63)\end{array}$ & $\begin{array}{c}0.77(0.34 \\
1.75)\end{array}$ & $\begin{array}{c}1.19(0.54 \\
2.62)\end{array}$ & \\
\hline
\end{tabular}

1. The cells in blue are results of pair-wise meta-analysis and the column treatment is compared with the row treatment. The results are presented by hazard ratio (HR) and $95 \%$ credible interval (CrI). The cells in red are results of network meta-analysis and the row treatment is compared with the column treatment. The results are presented by hazard ratio (HR) and $95 \%$ credible interval $(\mathrm{CrI})$.

2. TACE: transcatheter arterial chemoembolization; EBRT: external-beam radiation therapy; HIFU: high intensity focused ultrasound; PEI: percutaneous ethanol injection; RFA: radiofrequency ablation; RT: radiotherapy; SOR: sorafenib; TARE-90Y: yttrium-90 radioembolization; DEB-TACE: drug-eluting beads-transcatheter arterial chemoembolization; PAI: percutaneous acetic acid injection.

TACE+RT were associated with an increase in OS-2 compared to those treated with TACE+PEI $(\mathrm{HR}<1,95 \%$ CrI excludes 1). TARE - 90Y, PEI, RFA and RT appeared to be less effective than TACE + RFA (HR $>1,95 \% \mathrm{CrI}$ excludes 1).

For OS - 3 (Table 4), TACE + HIFU, TACE + RFA, $\mathrm{TACE}+\mathrm{RT}$ and DEB-TACE were associated with an increased OS-3 in comparison to its counterpart TACE (HR $<1,95 \%$ CrI excludes 1$)$. PEI was less effective than TACE + HIFU (HR $=3.47,95 \%$ CrI, 1.37 - 8.76) and both PEI and RFA appeared to be less effective than TACE + RFA $(\mathrm{HR}=2.75$ 95\%, CrI: $1.73-4.37$; HR $=1.57$ 95\%, CrI: 1.15 - 2.15). TARE - 90Y, PAI, PEI, RFA and RT were less effective than TACE + RT (HR $>1,95 \% \mathrm{CrI}$ excludes 1).

Comparisons among therapies with respect to OS-4 were displayed in Table 5. Therapies including TACE + EBRT, TACE + HIFU, TACE + RFA, TACE + RT, TACE + SOR, TARE - 90Y and RFA were superior to TACE monotherapy (all $\mathrm{HR}<1,95 \%$ CrI excludes 1). TACE + PEI, TACE + RT, TARE - 90Y, DEB - TACE, PEI, RFA and RT were less effective than TACE + EBRT (HR > $1,95 \% \mathrm{CrI}$ excludes 1 ). A few therapies appeared to be less effective than TACE + HIFU including TACE + PEI, TACE + RFA, TACE + SOR, TARE - 90Y, DEB - TACE, PEI, RFA and RT (HR $>1,95 \%$ CrI excludes 1$)$. TACE + RFA, TACE + RT, TACE + SOR, TARE - 90Y and RFA exhibited enhanced efficacy compared to TACE + PEI (HR $<1,95 \%$ CrI excludes 1). TACE + SOR, TARE 90Y, DEB - TACE, PEI, RFA and RT were less effective than TACE + RFA $(\mathrm{HR}>1,95 \%$ CrI excludes 1$)$.

Since the reliability of network meta-analysis can be assessed by consistency between direct and indirect evidence, we also obtained a net heat plot to achieve that purpose (Figure 2). The horizontal and vertical axis corresponds to evidence of study designs obtained from direct and indirect comparisons, respectively. The size of the square indicates the contribution of direct estimates 
Table 3: Comparing the relative efficacy of interventions with respect to two-year survival status using pairwise and network meta-analysis

\begin{tabular}{|c|c|c|c|c|c|c|c|c|c|c|c|c|c|}
\hline & TACE & $\begin{array}{c}\text { TACE } \\
+ \text { EBRT }\end{array}$ & $\begin{array}{c}\text { TACE } \\
+ \text { HIFU }\end{array}$ & $\begin{array}{l}\text { TACE } \\
+ \text { PEI }\end{array}$ & $\begin{array}{l}\text { TACE } \\
+ \text { RFA }\end{array}$ & $\begin{array}{c}\text { TACE } \\
+\mathrm{RT}\end{array}$ & $\begin{array}{l}\text { TACE } \\
+ \text { SOR }\end{array}$ & $\begin{array}{l}\text { TARE } \\
-90 Y\end{array}$ & $\begin{array}{c}\text { DEB } \\
\text {-TACE }\end{array}$ & PAI & PEI & RFA & RT \\
\hline TACE & & $\begin{array}{c}0.71(0.59 \\
0.83)\end{array}$ & $\begin{array}{c}0.25(0.22 \\
0.28)\end{array}$ & $\begin{array}{c}0.51(0.46 \\
0.56)\end{array}$ & $\begin{array}{c}0.53(0.42 \\
0.63)\end{array}$ & $\begin{array}{c}0.53(0.47 \\
0.59)\end{array}$ & $\begin{array}{c}0.55(0.50 \\
0.60)\end{array}$ & $\begin{array}{c}0.87(0.80 \\
0.94)\end{array}$ & $\begin{array}{c}0.52(0.47 \\
0.57)\end{array}$ & - & - & $\begin{array}{c}0.90(0.80 \\
0.99)\end{array}$ & $\begin{array}{c}0.44(0.34 \\
0.54)\end{array}$ \\
\hline TACE+EBRT & $\begin{array}{c}0.71(0.33 \\
1.53)\end{array}$ & & - & - & - & - & - & - & - & - & - & - & - \\
\hline TACE+HIFU & $\begin{array}{c}0.31(0.18 \\
0.54)\end{array}$ & $\begin{array}{c}0.44(0.17 \\
1.13)\end{array}$ & & - & - & - & - & - & - & - & - & - & - \\
\hline TACE+PEI & $\begin{array}{c}0.76(0.55 \\
1.04)\end{array}$ & $\begin{array}{c}1.07(0.46 \\
2.45)\end{array}$ & $\begin{array}{c}2.41(1.28 \\
4.53)\end{array}$ & & - & - & - & - & - & - & $\begin{array}{c}2.02(1.77 \\
2.35)\end{array}$ & - & - \\
\hline TACE+RFA & $\begin{array}{c}0.46(0.31 \\
0.67)\end{array}$ & $\begin{array}{c}0.64(0.27 \\
1.52)\end{array}$ & $\begin{array}{c}1.45(0.75 \\
2.83)\end{array}$ & $\begin{array}{c}0.60(0.38 \\
0.97)\end{array}$ & & - & - & - & - & - & - & $\begin{array}{c}1.90(1.66, \\
2.22)\end{array}$ & - \\
\hline TACE+RT & $\begin{array}{c}0.44(0.29 \\
0.67)\end{array}$ & $\begin{array}{c}0.62(0.26 \\
1.49)\end{array}$ & $\begin{array}{c}1.40(0.71 \\
2.78)\end{array}$ & $\begin{array}{c}0.58(0.34 \\
0.99)\end{array}$ & $\begin{array}{c}0.96(0.55 \\
1.70)\end{array}$ & & - & - & - & - & - & - & $\begin{array}{c}5.56(4.44, \\
7.41)\end{array}$ \\
\hline TACE+SOR & $\begin{array}{c}0.67(0.47 \\
0.95)\end{array}$ & $\begin{array}{c}0.94(0.40 \\
2.18)\end{array}$ & $\begin{array}{c}2.12(1.11 \\
4.05)\end{array}$ & $\begin{array}{c}0.88(0.55 \\
1.42)\end{array}$ & $\begin{array}{c}1.46(0.87 \\
2.46)\end{array}$ & $\begin{array}{c}1.52(0.88 \\
2.61)\end{array}$ & & - & - & - & - & - & - \\
\hline TARE-90Y & $\begin{array}{c}0.87(0.59 \\
1.28)\end{array}$ & $\begin{array}{c}1.23(0.52 \\
2.90)\end{array}$ & $\begin{array}{c}2.78(1.43 \\
5.40)\end{array}$ & $\begin{array}{c}1.15(0.70 \\
1.90)\end{array}$ & $\begin{array}{c}1.91(1.11, \\
3.29)\end{array}$ & $\begin{array}{c}1.98(1.12 \\
3.50)\end{array}$ & $\begin{array}{c}1.31(0.78 \\
2.20)\end{array}$ & & - & - & - & - & - \\
\hline DEB-TACE & $\begin{array}{c}0.62(0.46 \\
0.84)\end{array}$ & $\begin{array}{c}0.87(0.38 \\
2.00)\end{array}$ & $\begin{array}{c}1.98(1.06 \\
3.68)\end{array}$ & $\begin{array}{c}0.82(0.53 \\
1.27)\end{array}$ & $\begin{array}{c}1.36(0.83 \\
2.22)\end{array}$ & $\begin{array}{c}1.41(0.84 \\
2.36)\end{array}$ & $\begin{array}{c}0.93(0.59 \\
1.47)\end{array}$ & $\begin{array}{c}0.71(0.44, \\
1.16)\end{array}$ & & - & - & - & - \\
\hline PAI & $\begin{array}{c}1.12(0.46 \\
2.70)\end{array}$ & $\begin{array}{c}1.58(0.49 \\
5.07)\end{array}$ & $\begin{array}{c}3.56(1.26 \\
10.03)\end{array}$ & $\begin{array}{c}1.48(0.60 \\
3.63)\end{array}$ & $\begin{array}{c}2.45(0.99 \\
6.04)\end{array}$ & $\begin{array}{c}2.54(0.96 \\
6.74)\end{array}$ & $\begin{array}{c}1.68(0.65, \\
4.33)\end{array}$ & $\begin{array}{c}1.28(0.49 \\
3.35)\end{array}$ & $\begin{array}{c}1.80(0.71 \\
4.57)\end{array}$ & & $\begin{array}{c}1.78(0.93, \\
1.60)\end{array}$ & - & - \\
\hline PEI & $\begin{array}{c}1.32(0.90 \\
1.93)\end{array}$ & $\begin{array}{c}1.85(0.79 \\
4.37)\end{array}$ & $\begin{array}{c}4.19(2.16 \\
8.13)\end{array}$ & $\begin{array}{c}1.74(1.15 \\
2.64)\end{array}$ & $\begin{array}{c}2.88(1.88 \\
4.41)\end{array}$ & $\begin{array}{c}2.99(1.70 \\
5.26)\end{array}$ & $\begin{array}{c}1.97(1.18 \\
3.31)\end{array}$ & $\begin{array}{c}1.51(0.88 \\
2.59)\end{array}$ & $\begin{array}{l}2.12(1.31 \\
\quad 3.44)\end{array}$ & $\begin{array}{c}1.18(0.53, \\
2.60)\end{array}$ & & $\begin{array}{c}0.43(0.35 \\
0.50)\end{array}$ & - \\
\hline RFA & $\begin{array}{c}0.76(0.53 \\
1.08)\end{array}$ & $\begin{array}{c}1.07(0.46 \\
2.49)\end{array}$ & $\begin{array}{c}2.42(1.27 \\
4.62)\end{array}$ & $\begin{array}{c}1.00(0.65 \\
1.55)\end{array}$ & $\begin{array}{c}1.66(1.21, \\
2.28)\end{array}$ & $\begin{array}{c}1.73(1.00 \\
2.98)\end{array}$ & $\begin{array}{c}1.14(0.69 \\
1.87)\end{array}$ & $\begin{array}{c}0.87(0.52 \\
1.47)\end{array}$ & $\begin{array}{c}1.22(0.77 \\
1.94)\end{array}$ & $\begin{array}{c}0.68(0.29 \\
1.61)\end{array}$ & $\begin{array}{c}0.58(0.42 \\
0.80)\end{array}$ & & - \\
\hline RT & $\begin{array}{c}1.03(0.57 \\
1.86)\end{array}$ & $\begin{array}{c}1.45(0.55 \\
3.82)\end{array}$ & $\begin{array}{c}3.27(1.47 \\
7.32)\end{array}$ & $\begin{array}{c}1.36(0.69 \\
2.67)\end{array}$ & $\begin{array}{c}2.25(1.11 \\
4.57)\end{array}$ & $\begin{array}{c}2.34(1.29 \\
4.23)\end{array}$ & $\begin{array}{c}1.54(0.77 \\
3.07)\end{array}$ & $\begin{array}{c}1.18(0.58 \\
2.39)\end{array}$ & $\begin{array}{c}1.65(0.85 \\
3.21)\end{array}$ & $\begin{array}{c}0.92(0.32, \\
2.66)\end{array}$ & $\begin{array}{c}0.78(0.39 \\
1.58)\end{array}$ & $\begin{array}{c}1.35(0.68 \\
2.70)\end{array}$ & \\
\hline
\end{tabular}

1. The cells in blue are results of pair-wise meta-analysis and the column treatment is compared with the row treatment. The results are presented by hazard ratio (HR) and $95 \%$ credible interval $(\mathrm{CrI})$. The cells in red are results of network meta-analysis and the row treatment is compared with the column treatment. The results are presented by hazard ratio (HR) and $95 \%$ credible interval (CrI).

2. TACE: transcatheter arterial chemoembolization; EBRT: external-beam radiation therapy; HIFU: high intensity focused ultrasound; PEI: percutaneous ethanol injection; RFA: radiofrequency ablation; RT: radiotherapy; SOR: sorafenib; TARE-90Y: yttrium-90 radioembolization; DEB-TACE: drug-eluting beads-transcatheter arterial

chemoembolization; PAI: percutaneous acetic acid injection.

in a specific design in relation to the network estimate in the corresponding design. In other words, the size of square suggests the extent of direct estimates of study designs contributed to the corresponding mixed estimates. In Figure 2A, designs including TACE: PEI, TACE: TACE + PEI, TACE + RFA: RFA and TACE: TACE + RT had substantial effects on the corresponding network estimates. The corresponding colors in the net heat plot reveal changes in inconsistency between direct and indirect evidence in a study design once direct evidence of this design is detached. Blue colors suggest an increase in inconsistency whereas warm colors suggest a decrease in inconsistency. The strongest reduction in inconsistency resulted from the detachment of studies TACE: TACE + RT, TACE: RT, TACE: PEI and TACE + RT: RT since they appeared to have the most intensive colors (Figure 2A). This pattern was also replicated in Figure $2 \mathrm{~B}$ to $2 \mathrm{D}$. Therefore, study designs corresponding to TACE: TACE + RT, TACE: RT and TACE + RT: RT appeared to have strong inconsistency between direct and indirect evidence since there was significant reduction in inconsistence once we detached the corresponding direct evidence of these studies.

Finally, Figure 3 provides the ranking probabilities of these interventions based on their corresponding SUCRA values. TACE + HIFU exhibited the most promising result whereas PEI has the least efficacy with respect to OS-1 year (Figure 3A). Similar rankings were displayed in Figure 3B-3D, revealing that TACE + HIFU had largest efficacy whereas PEI was the least efficacious one. Moreover, combined treatments including TACE + RFA and TACE + RT provided patients with almost equivalent efficacy in comparison to TACE + HIFU. Aside from that, introducing other therapies (EBRT, HIFU, RFA, RT, and SOR) into TACE substantially enhanced the efficacy of TACE

\section{DISCUSSION}

TACE has been widely introduced to HCC patients when surgical resection is believed to be inappropriate [59]. Some detractors, however, have voiced that TACE 
Table 4: Comparing the relative efficacy of interventions with respect to three-year survival status using pairwise and network meta-analysis

\begin{tabular}{|c|c|c|c|c|c|c|c|c|c|c|c|c|c|}
\hline & TACE & $\begin{array}{c}\text { TACE } \\
+ \text { EBRT }\end{array}$ & $\begin{array}{c}\text { TACE } \\
+ \text { HIFU }\end{array}$ & $\begin{array}{l}\text { TACE } \\
+ \text { PEI }\end{array}$ & $\begin{array}{l}\text { TACE } \\
+ \text { RFA }\end{array}$ & $\begin{array}{c}\text { TACE } \\
+ \text { RT }\end{array}$ & $\begin{array}{l}\text { TACE } \\
+ \text { SOR }\end{array}$ & $\begin{array}{l}\text { TARE } \\
-90 Y\end{array}$ & $\begin{array}{c}\text { DEB } \\
\text {-TACE }\end{array}$ & PAI & PEI & RFA & RT \\
\hline TACE & & $\begin{array}{c}0.69(0.59 \\
0.80)\end{array}$ & $\begin{array}{c}0.41(0.35 \\
0.48)\end{array}$ & $\begin{array}{c}0.50(0.43 \\
0.56)\end{array}$ & $\begin{array}{c}0.56(0.46 \\
0.65)\end{array}$ & $\begin{array}{c}0.54(0.48 \\
0.60)\end{array}$ & $\begin{array}{c}0.64(0.56 \\
0.72)\end{array}$ & $\begin{array}{c}0.84(0.77 \\
0.90)\end{array}$ & $\begin{array}{c}0.50(0.44 \\
0.56)\end{array}$ & - & - & $\begin{array}{c}0.80(0.69 \\
0.91)\end{array}$ & $\begin{array}{c}0.44(0.34 \\
0.54)\end{array}$ \\
\hline TACE+EBRT & $\begin{array}{c}0.69(0.32 \\
1.50)\end{array}$ & & - & - & - & - & - & - & - & - & - & - & - \\
\hline TACE+HIFU & $\begin{array}{c}0.41(0.19 \\
0.90)\end{array}$ & $\begin{array}{c}0.59(0.20 \\
1.79)\end{array}$ & & - & - & - & - & - & - & - & - & - & - \\
\hline TACE+PEI & $\begin{array}{c}0.77(0.53 \\
1.13)\end{array}$ & $\begin{array}{c}1.12(0.47 \\
2.67)\end{array}$ & $\begin{array}{c}1.89(0.79 \\
4.50)\end{array}$ & & - & - & - & - & - & - & $\begin{array}{c}1.39(0.82 \\
4.55)\end{array}$ & - & - \\
\hline TACE+RFA & $\begin{array}{c}0.52(0.35 \\
0.78)\end{array}$ & $\begin{array}{c}0.75(0.31 \\
1.81)\end{array}$ & $\begin{array}{c}1.26(0.52 \\
3.04)\end{array}$ & $\begin{array}{c}0.67(0.39 \\
1.13)\end{array}$ & & - & - & - & - & - & - & $\begin{array}{c}1.76(1.56 \\
2.00)\end{array}$ & - \\
\hline TACE+RT & $\begin{array}{c}0.45(0.29 \\
0.69)\end{array}$ & $\begin{array}{c}0.65(0.27 \\
1.58)\end{array}$ & $\begin{array}{c}1.09(0.45 \\
2.66)\end{array}$ & $\begin{array}{c}0.58(0.33 \\
1.02)\end{array}$ & $\begin{array}{c}0.87(0.48 \\
1.56)\end{array}$ & & - & - & - & - & - & - & $\begin{array}{c}5.56(4.44, \\
7.41)\end{array}$ \\
\hline TACE+SOR & $\begin{array}{c}0.71(0.45 \\
1.13)\end{array}$ & $\begin{array}{c}1.04(0.42 \\
2.56)\end{array}$ & $\begin{array}{c}1.74(0.71 \\
4.31)\end{array}$ & $\begin{array}{c}0.92(0.51 \\
1.67)\end{array}$ & $\begin{array}{c}1.38(0.75 \\
2.54)\end{array}$ & $\begin{array}{c}1.60(0.85, \\
2.98)\end{array}$ & & - & - & - & - & - & - \\
\hline TARE-90Y & $\begin{array}{c}0.84(0.57 \\
1.25)\end{array}$ & $\begin{array}{c}1.22(0.51 \\
2.93)\end{array}$ & $\begin{array}{c}2.06(0.86, \\
4.93)\end{array}$ & $\begin{array}{c}1.09(0.63 \\
1.88)\end{array}$ & $\begin{array}{c}1.63(0.93 \\
2.86)\end{array}$ & $\begin{array}{c}1.89(1.06, \\
3.36)\end{array}$ & $\begin{array}{c}1.18(0.65, \\
2.16)\end{array}$ & & - & - & - & - & - \\
\hline DEB-TACE & $\begin{array}{c}0.64(0.46 \\
0.89)\end{array}$ & $\begin{array}{c}0.93(0.40 \\
2.16)\end{array}$ & $\begin{array}{c}1.56(0.67 \\
3.64)\end{array}$ & $\begin{array}{c}0.83(0.50 \\
1.36)\end{array}$ & $\begin{array}{c}1.23(0.73 \\
2.08)\end{array}$ & $\begin{array}{c}1.43(0.83 \\
2.44)\end{array}$ & $\begin{array}{c}0.89(0.51 \\
1.57)\end{array}$ & $\begin{array}{c}0.76(0.45 \\
1.26)\end{array}$ & & - & - & - & - \\
\hline PAI & $\begin{array}{c}1.25(0.49 \\
3.20)\end{array}$ & $\begin{array}{c}1.81(0.53 \\
6.14)\end{array}$ & $\begin{array}{c}3.05(0.90 \\
10.35)\end{array}$ & $\begin{array}{c}1.62(0.61 \\
4.30)\end{array}$ & $\begin{array}{c}2.42(0.96 \\
6.07)\end{array}$ & $\begin{array}{c}2.79(1.00, \\
7.83)\end{array}$ & $\begin{array}{c}1.75(0.62, \\
4.97)\end{array}$ & $\begin{array}{c}1.48(0.54 \\
4.09)\end{array}$ & $\begin{array}{c}1.96(0.72 \\
5.29)\end{array}$ & & $\begin{array}{c}1.14(0.93 \\
1.45)\end{array}$ & - & - \\
\hline PEI & $\begin{array}{c}1.42(0.86, \\
2.34)\end{array}$ & $\begin{array}{c}2.06(0.82 \\
5.20)\end{array}$ & $\begin{array}{c}3.47(1.37, \\
8.76)\end{array}$ & $\begin{array}{c}1.84(1.04 \\
3.24)\end{array}$ & $\begin{array}{c}2.75(1.73 \\
4.37)\end{array}$ & $\begin{array}{c}3.17(1.65, \\
6.11)\end{array}$ & $\begin{array}{c}1.99(1.01, \\
3.91)\end{array}$ & $\begin{array}{c}1.68(0.89, \\
3.17)\end{array}$ & $\begin{array}{c}2.22(1.22 \\
4.04)\end{array}$ & $\begin{array}{c}1.14(0.51 \\
2.52)\end{array}$ & & $\begin{array}{c}0.48(0.41, \\
0.55)\end{array}$ & - \\
\hline RFA & $\begin{array}{c}0.81(0.55 \\
1.21)\end{array}$ & $\begin{array}{c}1.18(0.49 \\
2.83)\end{array}$ & $\begin{array}{c}1.99(0.83 \\
4.77)\end{array}$ & $\begin{array}{c}1.05(0.63 \\
1.75)\end{array}$ & $\begin{array}{c}1.57(1.15 \\
2.15)\end{array}$ & $\begin{array}{c}1.82(1.02 \\
3.25)\end{array}$ & $\begin{array}{c}1.14(0.62 \\
2.09)\end{array}$ & $\begin{array}{c}0.96(0.55 \\
1.68)\end{array}$ & $\begin{array}{c}1.27(0.76 \\
2.13)\end{array}$ & $\begin{array}{c}0.65(0.27 \\
1.56)\end{array}$ & $\begin{array}{c}0.57(0.40 \\
0.82)\end{array}$ & & - \\
\hline RT & $\begin{array}{c}1.04(0.57 \\
1.90)\end{array}$ & $\begin{array}{c}1.50(0.56 \\
4.03)\end{array}$ & $\begin{array}{c}2.53(0.94 \\
6.79)\end{array}$ & $\begin{array}{c}1.34(0.66 \\
2.73)\end{array}$ & $\begin{array}{c}2.00(0.97 \\
4.15)\end{array}$ & $\begin{array}{c}2.32(1.26, \\
4.24)\end{array}$ & $\begin{array}{c}1.45(0.68 \\
3.10)\end{array}$ & $\begin{array}{c}1.23(0.60 \\
2.52)\end{array}$ & $\begin{array}{c}1.62(0.82 \\
3.23)\end{array}$ & $\begin{array}{c}0.83(0.27 \\
2.53)\end{array}$ & $\begin{array}{c}0.73(0.33 \\
1.60)\end{array}$ & $\begin{array}{c}1.27(0.62 \\
2.62)\end{array}$ & \\
\hline
\end{tabular}

1. The cells in blue are results of pair-wise meta-analysis and the column treatment is compared with the row treatment. The results are presented by hazard ratio (HR) and $95 \%$ credible interval (CrI). The cells in red are results of network meta-analysis and the row treatment is compared with the column treatment. The results are presented by hazard ratio (HR) and $95 \%$ credible interval (CrI).

2. TACE: transcatheter arterial chemoembolization; EBRT: external-beam radiation therapy; HIFU: high intensity focused ultrasound; PEI: percutaneous ethanol injection; RFA: radiofrequency ablation; RT: radiotherapy; SOR: sorafenib; TARE-90Y: yttrium-90 radioembolization; DEB-TACE: drug-eluting beads-transcatheter arterial

chemoembolization; PAI: percutaneous acetic acid injection.

should not be carried out for patients with portal vein tumor thrombus (PVTT) as it may result in ischemic liver damage under certain circumstances [60]. As suggested by both the pair-wise meta-analysis and network metaanalysis in our study, TACE + HIFU appeared to be far more effective than TACE monotherapy over the fouryear period. HIFU is usually combined with TACE in order to induce complete tumor necrosis which is not achievable by using TACE monotherapy [14]. A prospective randomized trial indicated that patients who underwent TACE + HIFU exhibited significantly better responses than those who underwent repeated segmental TACE monotherapy [56]. HIFU ablation is an effective therapy which is characterized by non-invasiveness and it is particularly suited for treating localized tumors [61]. The combined therapy of HIFU + TACE reduced the side effects resulted from the use of repeated TACE without significant efficacy loss.

In our study, the combined therapy of TACE and RFA also exhibited promising results and an average of $50 \%$ reduction in the HR was suggested in comparison to those with TACE. Two common combined approaches have been introduced into clinical practices. The first approach is commonly carried out by firstly implementing TACE followed by RFA. The second approach is conducted in the reverse order. Choosing an appropriate time interval between TACE and RFA also has a significant impact on the efficacy and safety [62]. For instance, liver functions are more likely to be preserved if sufficient time is allowed between the implementation of TACE and RFA [63]. However, an extended time between TACE and RFA may increase the number of treatment days along with the associated administration costs. On the other hand, a relatively short interval can contribute to stronger efficacy as the synergistic effects induced by TACE and RFA are likely to be increased. Nevertheless, such an increase in the synergistic effects may also be associated with a potential increase in the risk of liver dysfunction which is commonly observed in cirrhotic patients [62]. As a result, the sequence in which TACE and RFA are carried out as well as the optimal interval between these therapies 
Table 5: Comparing the relative efficacy of therapies with respect to four-year survival status using pairwise and network meta-analysis

\begin{tabular}{|c|c|c|c|c|c|c|c|c|c|c|c|c|}
\hline & TACE & $\begin{array}{c}\text { TACE } \\
+ \text { EBRT }\end{array}$ & $\begin{array}{c}\text { TACE } \\
+ \text { HIFU }\end{array}$ & $\begin{array}{l}\text { TACE } \\
+ \text { PEI }\end{array}$ & $\begin{array}{l}\text { TACE } \\
+ \text { RFA }\end{array}$ & $\begin{array}{c}\text { TACE } \\
+ \text { RT }\end{array}$ & $\begin{array}{l}\text { TACE } \\
+ \text { SOR }\end{array}$ & $\begin{array}{l}\text { TARE } \\
-90 Y\end{array}$ & $\begin{array}{c}\text { DEB } \\
\text {-TACE }\end{array}$ & PEI & RFA & RT \\
\hline TACE & & $\begin{array}{c}0.69(0.59 \\
0.80)\end{array}$ & $\begin{array}{c}0.41(0.35 \\
0.48)\end{array}$ & $\begin{array}{c}1.29(0.92 \\
1.66)\end{array}$ & $\begin{array}{c}0.60(0.50 \\
0.69)\end{array}$ & $\begin{array}{c}0.55(0.49 \\
0.60)\end{array}$ & $\begin{array}{c}0.67(0.57 \\
0.76)\end{array}$ & $\begin{array}{c}0.86(0.79 \\
0.93)\end{array}$ & $\begin{array}{c}0.94(0.74 \\
1.14)\end{array}$ & - & - & $\begin{array}{c}0.44(0.34 \\
0.54)\end{array}$ \\
\hline TACE+EBRT & $\begin{array}{c}0.69(0.59 \\
0.80)\end{array}$ & & - & - & - & - & - & - & - & - & - & - \\
\hline TACE + HIFU & $\begin{array}{c}0.41(0.35 \\
0.48)\end{array}$ & $\begin{array}{c}0.59(0.48 \\
0.74)\end{array}$ & & - & - & - & - & - & - & - & - & - \\
\hline TACE+PEI & $\begin{array}{c}1.19(0.91 \\
1.54)\end{array}$ & $\begin{array}{c}1.72(1.27 \\
2.33)\end{array}$ & $\begin{array}{c}2.90(2.13 \\
3.93)\end{array}$ & & - & - & - & - & - & $\begin{array}{c}1.39(0.82 \\
4.54)\end{array}$ & - & - \\
\hline TACE+RFA & $\begin{array}{c}0.58(0.51 \\
0.65)\end{array}$ & $\begin{array}{c}0.84(0.69 \\
1.01)\end{array}$ & $\begin{array}{c}1.41(1.16 \\
1.71)\end{array}$ & $\begin{array}{c}0.49(0.37 \\
0.64)\end{array}$ & & - & - & - & - & - & $\begin{array}{c}1.59(1.43 \\
1.79)\end{array}$ & - \\
\hline TACE+RT & $\begin{array}{c}0.47(0.43 \\
0.52)\end{array}$ & $\begin{array}{c}0.68(0.57 \\
0.82)\end{array}$ & $\begin{array}{c}1.15(0.95 \\
1.38)\end{array}$ & $\begin{array}{c}0.40(0.30 \\
0.52)\end{array}$ & $\begin{array}{c}0.81(0.70 \\
0.95)\end{array}$ & & - & - & - & - & - & $\begin{array}{c}5.56(4.44 \\
7.41)\end{array}$ \\
\hline TACE+SOR & $\begin{array}{c}0.77(0.67 \\
0.88)\end{array}$ & $\begin{array}{c}1.11(0.91 \\
1.36)\end{array}$ & $\begin{array}{c}1.87(1.52 \\
2.31)\end{array}$ & $\begin{array}{c}0.65(0.48 \\
0.87)\end{array}$ & $\begin{array}{c}1.33(1.11 \\
1.59)\end{array}$ & $\begin{array}{c}1.63(1.38 \\
1.93)\end{array}$ & & - & - & - & - & - \\
\hline TARE-90Y & $\begin{array}{c}0.89(0.82 \\
0.96)\end{array}$ & $\begin{array}{c}1.29(1.08 \\
1.53)\end{array}$ & $\begin{array}{c}2.17(1.82 \\
2.59)\end{array}$ & $\begin{array}{c}0.75(0.57 \\
0.98)\end{array}$ & $\begin{array}{c}1.54(1.33 \\
1.77)\end{array}$ & $\begin{array}{c}1.89(1.66 \\
2.15)\end{array}$ & $\begin{array}{c}1.16(0.99 \\
1.36)\end{array}$ & & - & - & - & - \\
\hline DEB-TACE & $\begin{array}{c}0.98(0.79 \\
1.20)\end{array}$ & $\begin{array}{c}1.42(1.09 \\
1.83)\end{array}$ & $\begin{array}{c}2.38(1.83 \\
3.10)\end{array}$ & $\begin{array}{c}0.82(0.59 \\
1.15)\end{array}$ & $\begin{array}{c}1.69(1.33 \\
2.15)\end{array}$ & $\begin{array}{c}2.08(1.65 \\
2.62)\end{array}$ & $\begin{array}{c}1.27(0.99 \\
1.63)\end{array}$ & $\begin{array}{c}1.10(0.88 \\
1.38)\end{array}$ & & - & - & - \\
\hline PEI & $\begin{array}{c}1.08(0.88 \\
1.32)\end{array}$ & $\begin{array}{c}1.56(1.21 \\
2.01)\end{array}$ & $\begin{array}{c}2.63(2.04 \\
3.39)\end{array}$ & $\begin{array}{c}0.91(0.67 \\
1.24)\end{array}$ & $\begin{array}{c}1.86(1.53 \\
2.27)\end{array}$ & $\begin{array}{c}2.29(1.83 \\
2.87)\end{array}$ & $\begin{array}{c}1.40(1.10 \\
1.79)\end{array}$ & $\begin{array}{c}1.21(0.98 \\
1.51)\end{array}$ & $\begin{array}{c}1.10(0.83 \\
1.47)\end{array}$ & & $\begin{array}{c}0.80(0.65 \\
0.94)\end{array}$ & - \\
\hline RFA & $\begin{array}{c}0.85(0.77 \\
0.95)\end{array}$ & $\begin{array}{c}1.24(1.03 \\
1.49)\end{array}$ & $\begin{array}{c}2.08(1.72 \\
2.52)\end{array}$ & $\begin{array}{c}0.72(0.54 \\
0.95)\end{array}$ & $\begin{array}{c}1.47(1.34 \\
1.62)\end{array}$ & $\begin{array}{c}1.81(1.57 \\
2.10)\end{array}$ & $\begin{array}{c}1.11(0.93 \\
1.32)\end{array}$ & $\begin{array}{c}0.96(0.84 \\
1.10)\end{array}$ & $\begin{array}{c}0.87(0.69 \\
1.10)\end{array}$ & $\begin{array}{c}0.79(0.66 \\
0.94)\end{array}$ & & - \\
\hline RT & $\begin{array}{c}0.97(0.82 \\
1.15)\end{array}$ & $\begin{array}{c}1.40(1.11 \\
1.76)\end{array}$ & $\begin{array}{c}2.36(1.87 \\
2.98)\end{array}$ & $\begin{array}{c}0.81(0.60 \\
1.11)\end{array}$ & $\begin{array}{c}1.67(1.36 \\
2.06)\end{array}$ & $\begin{array}{c}2.06(1.73, \\
2.45)\end{array}$ & $\begin{array}{c}1.26(1.01 \\
1.57)\end{array}$ & $\begin{array}{c}1.09(0.90 \\
1.31)\end{array}$ & $\begin{array}{c}0.99(0.76 \\
1.30)\end{array}$ & $\begin{array}{c}0.90(0.69 \\
1.17)\end{array}$ & $\begin{array}{c}1.13(0.93 \\
1.39)\end{array}$ & \\
\hline
\end{tabular}

1. The cells in blue are results of pair-wise meta-analysis and the column treatment is compared with the row treatment. The results are presented by hazard ratio (HR) and $95 \%$ credible interval $(\mathrm{CrI})$. The cells in red are results of network meta-analysis and the row treatment is compared with the column treatment. The results are presented by hazard ratio (HR) and $95 \%$ credible interval (CrI).

2. TACE: transcatheter arterial chemoembolization; EBRT: external-beam radiation therapy; HIFU: high intensity focused ultrasound; PEI: percutaneous ethanol injection; RFA: radiofrequency ablation; RT: radiotherapy; SOR: sorafenib; TARE-90Y: yttrium-90 radioembolization; DEB-TACE: drug-eluting beads-transcatheter arterial

chemoembolization; PAI: percutaneous acetic acid injection.

must be determined before the implementation. Recently, some encouraging results have been obtained, indicating that TACE followed by RT is able to improve the survival status and the local response rate of unresectable HCC patients $[64,65]$. Several advantages of combining TACE with RT have been mentioned in the current literature. For instance, TACE may trigger the decrease of tumor volume and such a decrease may contribute to the increase in the radiation dose delivered to the target tumor [66].

On the other hand, our study indicated that PEI was considered to be the one with the least compelling results. The implementation of PEI is quite simple and it involves injecting ethanol into the tumor mass for inducing coagulative necrosis of neoplastic nodules. Unlike TACE, the side effects resulted from PEI is relatively small even if procedures are repeated several times. The less compelling results of PEI may be explained by the fact that the volume of ethanol injection plays a critical role in its efficacy and the corresponding volume of injection is determined by the maximum radius of the lesion [67]. In view of the advantages of the PEI and to enhance its efficacy, PEI combined with other interventions has been studied. For instance, incorporating TACE into PEI yielded a higher complete response rate and more favorable survival status [31, 68].

There are several limitations to be noted in our study. Firstly, we extracted survival data from individual studies and some data was estimated using the survival function if the original data was not available. Estimating survival data can be challenging, particularly the censoring status since it can only be obtained from the original data and the lack of censoring status will have a significant impact on the characteristics of the corresponding survival functions. Secondly, the sequence in which combined therapies were conducted may influence the results and our study did not allow us to assess the sequences due to the nature of the meta-analysis. The implementation interval between the two procedures and the volume of ethanol injection were rarely revealed from studies which prevented us to evaluate how these two potential factors were linked with efficacy and safety of the corresponding therapies.

Overall, this network meta-analysis concluded that pairwise combination of TACE + HIFU, TACE + RFA and TACE + RT exhibited strong efficacy for unresectable HCC patients whereas PEI seems to be less effective than other standard or combined therapies. 
(A) Overall Survival of 1 year

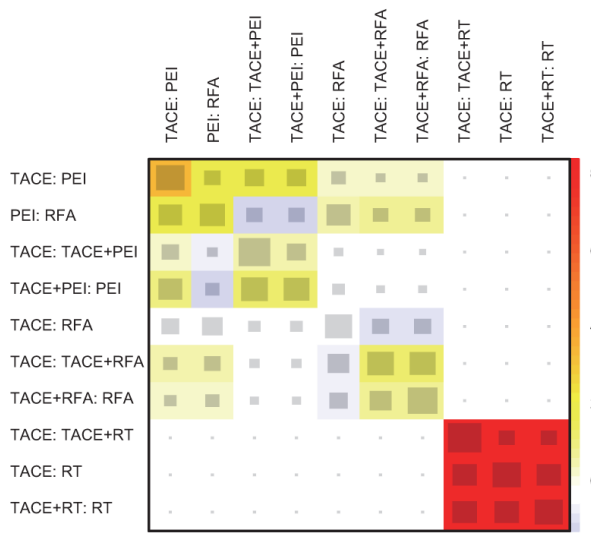

(C) Overall Survival of 3 years
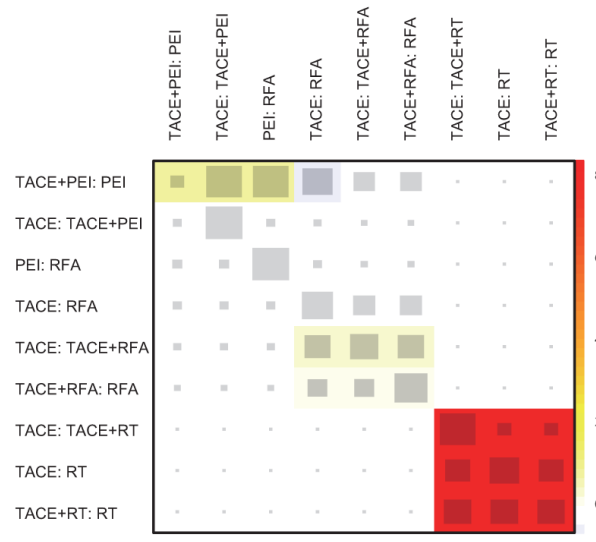

(B) Overall Survival of 2 years
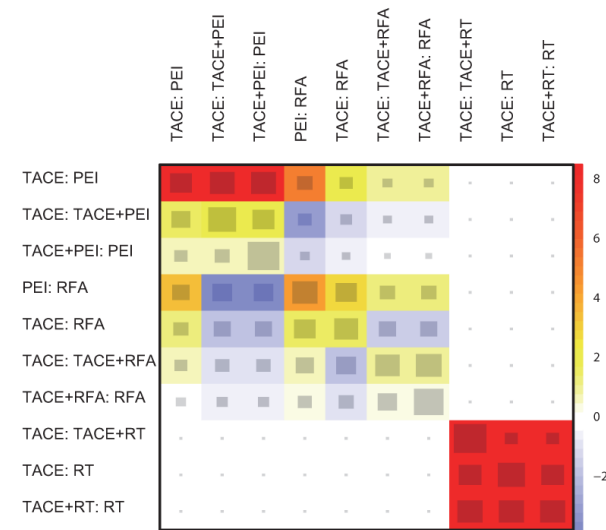

(D) Overall Survival of 4 years
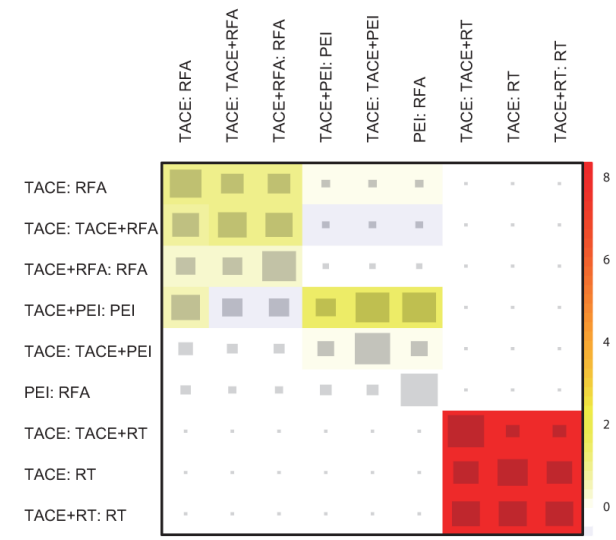

Figure 2: Net heat plots for different study designs revealing changes in inconsistency between direct and indirect evidence A. OS-1; B. OS-2; C. OS-3; D. OS-4.

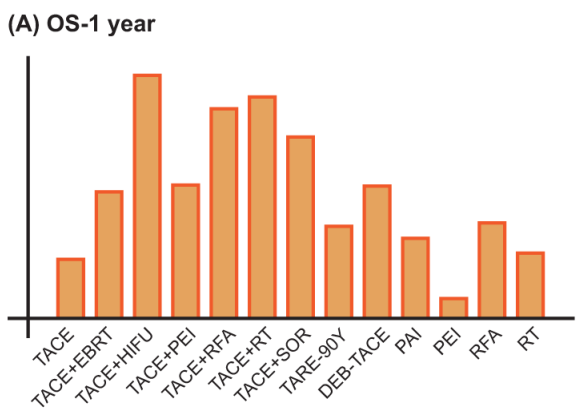

(B) OS-2 year

(C) OS-3 year
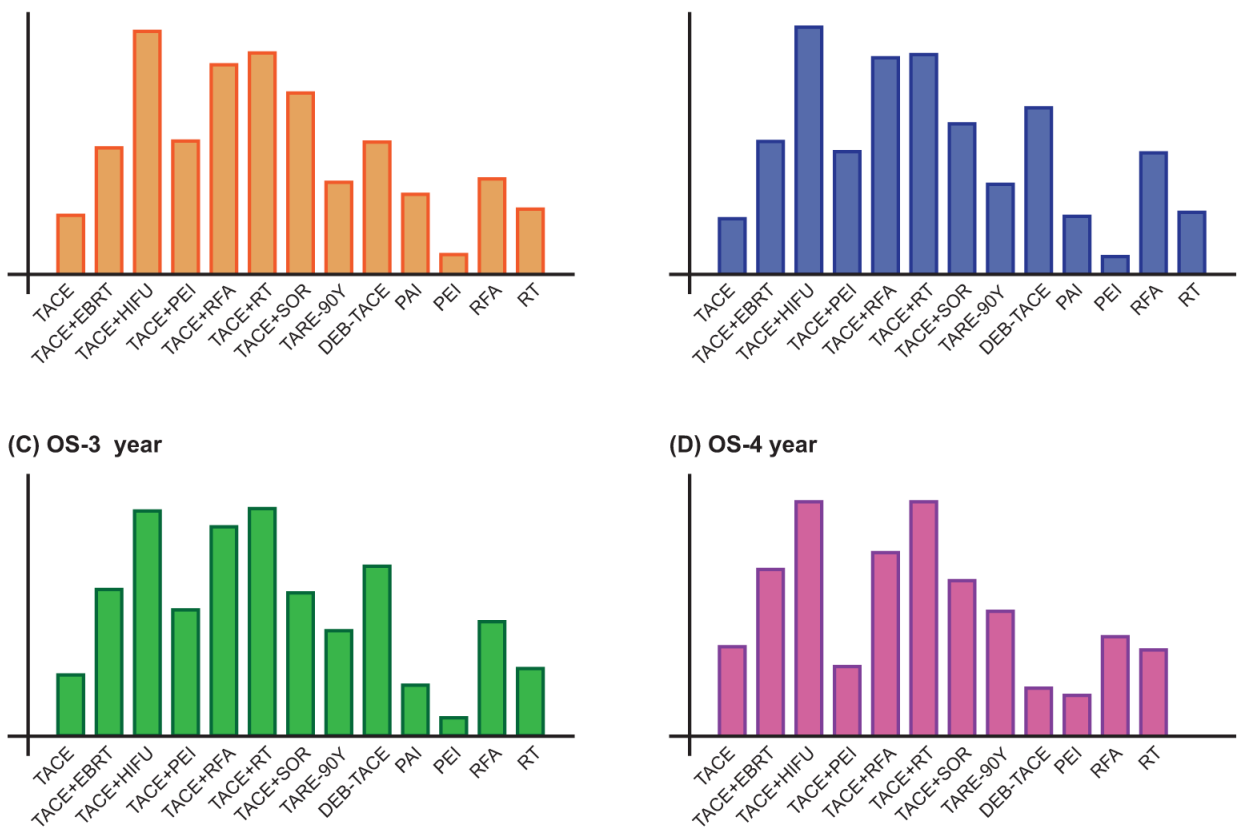

(D) OS-4 year

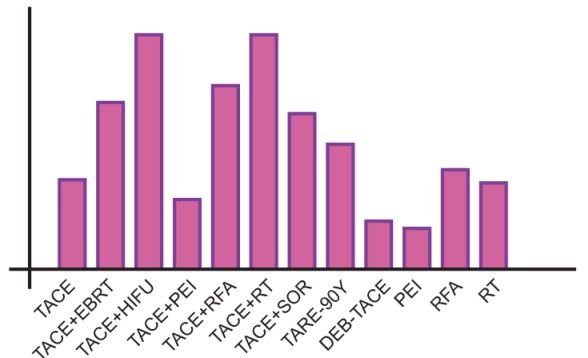

Figure 3: Surface under the cumulative ranking curve (SUCRA) of different interventions with respect to A. OS-1; B. OS-2; C. OS-3; and D. OS-4. 


\section{MATERIALS AND METHODS}

\section{Search strategy}

We searched for randomized control trials with the following search terms: "transcatheter arterial chemoembolization" (TACE) or "drug-eluting beadstrans -catheter arterial chemoembolization" (DEB-TACE) or "yttrium-90 radioembolization" (TARE-90Y) or "percutaneous ethanol injection" (PEI) or "radiofrequency ablation" (RFA) or "radiotherapy" (RT) or "percutaneous acetic acid injection" (PAI) or "high intensity focused ultrasound" (HIFU) or "external-beam radiation therapy" (EBRT ) or "sorafenib" (SOR) matched with "hepatocellular carcinoma" in PubMed, Embase, and China National Knowledge Internet (CNKI), which were updated on April 1, 2016, without restrictions on language. A manual search was also performed on the reference list of each relevant study. Previous meta-analyses and earlier reviews of intravesical instillation therapies in HCC were also reviewed to avoid any omissions. Individually, two reviewers performed the research and literature retrieval. All the arguments were solved with a mediating third reviewer.

\section{Inclusion and exclusion criteria}

Studies were eligible if: (1) were categorized as RCTs; (2) patients involved had HCC with a pathologic diagnosis and were over 18 years of age; (3) studies focused on the comparative efficacy of at least two standard or combined minimally invasive procedures (TACE, TACE + SOR, TACE + HIFU, TACE + PEI, DEB-TACE, TARE-90Y, TACE + EBRT, TACE + RT, TACE + RFA, RFA, PEI, PAI); (4) studies compared the efficacy between radiotherapy and minimally invasive procedures; (5) no surgical intervention had been performed on patients; (6) sufficient data were provided within the study. Articles were excluded if: (1) patients involved had multiple concurrent diseases, for instance, coronary artery disease as concomitance; (2) patients had received surgery, drugs, radiotherapy or other treatment approaches before study commencement; (3) there were no sufficient data provided by the study.

\section{Outcome measures and data extraction}

The following data were extracted from eligible studies: gender, patient age, sample size, duration of follow-up, and type of treatment. Two investigators reviewed the manuscripts of all the eligible studies and extracted data into a database independently. Other retrieved data included study duration, disease site, treatment protocols, study location and the number of patients in each arm. The efficacy of the corresponding therapies was measured by using multiple survival rates (overall survival of 1 year, OS-1; overall survival of 2 years, OS-2; overall survival of 3 years, OS-3; overall survival of 4 years, OS-4). Data extraction was conducted by two reviewers independently.

\section{Statistical analysis}

We initially carried out a conventional pair-wise meta-analysis which directly compares each pair of treatments. The corresponding hazard ratios (HRs) and $95 \%$ confidence intervals (CI) for each study were pooled in order to obtain the overall effect size. Furthermore, a network meta-analysis was performed for each endpoint with a Bayesian framework using $\mathrm{R}$ 3.2.3 software. Both direct and indirect evidence was synthesized in order to compare the efficacy which was assessed using the HRs and $95 \%$ credible intervals (CrI). Net heat plots were used to visually assess the degree of inconsistency between direct and indirect evidence. Then the cumulative ranking curve (SUCRA) was created to rank the standard or combined minimally invasive procedures with respect to their efficacy. The ranking probabilities were defined as cumulative probabilities that each therapy being ranked as the first, second and so on. For each endpoint, a therapy is more desirable than others if it has a larger SUCRA value.

\section{CONFLICTS OF INTEREST}

The authors declare no conflict of interest.

\section{REFERENCES}

1. Wang $\mathrm{X}$, Wang $\mathrm{Z}$ and $\mathrm{Wu} \mathrm{L}$. Combined measurements of tumor number and size helps estimate the outcome of resection of Barcelona clinic liver cancer stage B hepatocellular carcinoma. BMC surgery. 2016; 16:22.

2. Yang X, Guo Y, Du Y, Yang J, Li S, Liu S, Li K and Zhang D. Serum MicroRNA-21 as a Diagnostic Marker for Lung Carcinoma: A Systematic Review and Meta-Analysis. PloS one. 2014; 9:e97460.

3. Otaegui-Arrazola A, Amiano P, Elbusto A, Urdaneta E and Martinez-Lage P. Diet, cognition, and Alzheimer's disease: food for thought. European journal of nutrition. 2014; 53:1-23.

4. Lammer J, Malagari K, Vogl T, Pilleul F, Denys A, Watkinson A, Pitton M, Sergent G, Pfammatter T, Terraz S, Benhamou Y, Avajon Y, Gruenberger T, et al. Prospective randomized study of doxorubicin-eluting-bead embolization in the treatment of hepatocellular carcinoma: results of the PRECISION V study. Cardiovascular and interventional radiology. 2010; 33:41-52.

5. Bruix J, Sherman M and American Association for the Study of Liver D. Management of hepatocellular carcinoma: an update. Hepatology. 2011; 53:1020-1022.

6. Liu Z, Gao F, Yang G, Singh S, Lu M, Zhang T, Zhong Z, Zhang F and Tang R. Combination of radiofrequency 
ablation with transarterial chemoembolization for hepatocellular carcinoma: an up-to-date meta-analysis. Tumour biology. 2014; 35:7407-7413.

7. Lewandowski RJ, Kulik LM, Riaz A, Senthilnathan S, Mulcahy MF, Ryu RK, Ibrahim SM, Sato KT, Baker T, Miller FH, Omary R, Abecassis M and Salem R. A comparative analysis of transarterial downstaging for hepatocellular carcinoma: chemoembolization versus radioembolization. American journal of transplantation. 2009; 9:1920-1928.

8. Zhang Y, Li Y, Ji H, Zhao X and Lu H. Transarterial Y90 radioembolization versus chemoembolization for patients with hepatocellular carcinoma: A meta-analysis. Biosci Trends. 2015; 9:289-298.

9. Zhang T, Zhao YT, Wang Z, Li CR, Jin J, Jia AY, Wang SL, Song YW, Liu YP, Ren H, Fang H, Bao H, Liu XF, et al. Efficacy and Safety of Intensity-Modulated Radiotherapy Following Transarterial Chemoembolization in Patients With Unresectable Hepatocellular Carcinoma. Medicine (Baltimore). 2016; 95:e3789.

10. Huo TI, Huang YH and Wu JC. Percutaneous ablation therapy for hepatocellular carcinoma: current practice and future perspectives. J Chin Med Assoc. 2005; 68:155-159.

11. Cupino AC, Hair CD, Angle JF, Caldwell SH, Rich TA, Berg CL, Northup PG, Al-Osaimi AM and Argo CK. Does external beam radiation therapy improve survival following transarterial chemoembolization for unresectable hepatocellular carcinoma? Gastrointest Cancer Res. 2012; 5:13-17.

12. Murata S, Mine T, Sugihara F, Yasui D, Yamaguchi H, Ueda T, Onozawa S and Kumita S. Interventional treatment for unresectable hepatocellular carcinoma. World journal of gastroenterology. 2014; 20:13453-13465.

13. Kudo M, Ikeda M, Takayama T, Numata K, Izumi N, Furuse J, Okusaka T, Kadoya M, Yamashita S, Ito Y and Kokudo N. Safety and efficacy of sorafenib in Japanese patients with hepatocellular carcinoma in clinical practice: a subgroup analysis of GIDEON. J Gastroenterol. 2016.

14. Li C, Zhang W, Zhang R, Zhang L, Wu P and Zhang F. Therapeutic effects and prognostic factors in high-intensity focused ultrasound combined with chemoembolisation for larger hepatocellular carcinoma. European journal of cancer. 2010; 46:2513-2521.

15. Kirikoshi H, Saito S, Yoneda M, Fujita K, Mawatari H, Uchiyama T, Higurashi T, Goto A, Takahashi H, Abe Y, Inamori M, Kobayashi N, Kubota K, et al. Outcome of transarterial chemoembolization monotherapy, and in combination with percutaneous ethanol injection, or radiofrequency ablation therapy for hepatocellular carcinoma. Hepatology research. 2009; 39:553-562.

16. Meng MB, Cui YL, Lu Y, She B, Chen Y, Guan YS and Zhang RM. Transcatheter arterial chemoembolization in combination with radiotherapy for unresectable hepatocellular carcinoma: a systematic review and metaanalysis. Radiotherapy and oncology. 2009; 92:184-194.
17. Allgaier HP, Deibert P, Olschewski M, Spamer C, Blum U, Gerok W and Blum HE. Survival benefit of patients with inoperable hepatocellular carcinoma treated by a combination of transarterial chemoembolization and percutaneous ethanol injection--a single-center analysis including 132 patients. International journal of cancer. 1998; 79:601-605.

18. Bai W, Wang YJ, Zhao Y, Qi XS, Yin ZX, He CY, Li RJ, Wu KC, Xia JL, Fan DM and Han GH. Sorafenib in combination with transarterial chemoembolization improves the survival of patients with unresectable hepatocellular carcinoma: a propensity score matching study. Journal of digestive diseases. 2013; 14:181-190.

19. Bartolozzi C, Lencioni R, Caramella D, Vignali C, Cioni R, Mazzeo S, Carrai M, Maltinti G, Capria A and Conte PF. Treatment of large HCC: transcatheter arterial chemoembolization combined with percutaneous ethanol injection versus repeated transcatheter arterial chemoembolization. Radiology. 1995; 197:812-818.

20. Becker G, Soezgen T, Olschewski M, Laubenberger J, Blum HE and Allgaier HP. Combined TACE and PEI for palliative treatment of unresectable hepatocellular carcinoma. World journal of gastroenterology. 2005; 11:6104-6109.

21. Brunello F, Veltri A, Carucci P, Pagano E, Ciccone G, Moretto P, Sacchetto P, Gandini G and Rizzetto M. Radiofrequency ablation versus ethanol injection for early hepatocellular carcinoma: A randomized controlled trial. Scandinavian journal of gastroenterology. 2008; 43:727-735.

22. Carr BI, Kondragunta V, Buch SC and Branch RA. Therapeutic equivalence in survival for hepatic arterial chemoembolization and yttrium 90 microsphere treatments in unresectable hepatocellular carcinoma: a two-cohort study. Cancer. 2010; 116:1305-1314.

23. Cheng BQ, Jia CQ, Liu CT, Fan W, Wang QL, Zhang $\mathrm{ZL}$ and $\mathrm{Yi} \mathrm{CH}$. Chemoembolization combined with radiofrequency ablation for patients with hepatocellular carcinoma larger than $3 \mathrm{~cm}$ : a randomized controlled trial. Jama. 2008; 299:1669-1677.

24. Chia-Hsien Cheng J, Chuang VP, Cheng SH, Lin YM, Cheng TI, Yang PS, Jian JJ, You DL, Horng CF and Huang AT. Unresectable hepatocellular carcinoma treated with radiotherapy and/or chemoembolization. International journal of cancer. 2001; 96:243-252.

25. Dhanasekaran R, Kooby DA, Staley CA, Kauh JS, Khanna $\mathrm{V}$ and Kim HS. Comparison of conventional transarterial chemoembolization (TACE) and chemoembolization with doxorubicin drug eluting beads (DEB) for unresectable hepatocelluar carcinoma (HCC). Journal of surgical oncology. 2010; 101:476-480.

26. Ferrer Puchol MD, Parra C, Esteban E, Vano M, Forment M, Vera A and Cosin O. (2011). Comparison of doxorubicineluting bead transarterial chemoembolization (DEB-TACE) with conventional transarterial chemoembolization (TACE) for the treatment of hepatocellular carcinoma. [Spanish]. Radiologia, pp. 246-253. 
27. Guo WJ, Yu EX, Liu LM, Li J, Chen Z, Lin JH, Meng ZQ and Feng Y. Comparison between chemoembolization combined with radiotherapy and chemoembolization alone for large hepatocellular carcinoma. World journal of gastroenterology. 2003; 9:1697-1701.

28. Kamada K, Kitamoto M, Aikata H, Kawakami Y, Kono H, Imamura M, Nakanishi T and Chayama K. (2002). Combination of transcatheter arterial chemoembolization using cisplatin-lipiodol suspension and percutaneous ethanol injection for treatment of advanced small hepatocellular carcinoma. Am J Surg, pp. 284-290.

29. Kato T, Saito Y, Niwa M, Ishiguro J and Ogoshi K. (1994). Combination therapy of transcatheter chemoembolization and percutaneous ethanol injection therapy for unresectable hepatocellular carcinoma. Cancer Chemother Pharmacol, pp. S115-118.

30. Kim YC, Kim YH, Uhm SH, Seo YS, Park EK, Oh SY, Jeong E, Lee $\mathrm{S}$ and Choe JG. Radiation safety issues in Y-90 microsphere selective hepatic radioembolization therapy: Possible radiation exposure from the patients. Nuclear Medicine and Molecular Imaging. 2010; 44:252-260.

31. Koda M, Murawaki Y, Mitsuda A, Oyama K, Okamoto $\mathrm{K}$, Idobe $\mathrm{Y}$, Suou $\mathrm{T}$ and Kawasaki H. Combination therapy with transcatheter arterial chemoembolization and percutaneous ethanol injection compared with percutaneous ethanol injection alone for patients with small hepatocellular carcinoma: a randomized control study. Cancer. 2001; 92:1516-1524.

32. Kooby DA, Egnatashvili V, Srinivasan S, Chamsuddin A, Delman KA, Kauh J, Staley CA, 3rd and Kim HS. Comparison of yttrium-90 radioembolization and transcatheter arterial chemoembolization for the treatment of unresectable hepatocellular carcinoma. Journal of vascular and interventional radiology. 2010; 21:224-230.

33. Kudo M, Imanaka K, Chida N, Nakachi K, Tak WY, Takayama T, Yoon JH, Hori T, Kumada H, Hayashi N, Kaneko S, Tsubouchi H, Suh DJ, et al. Phase III study of sorafenib after transarterial chemoembolisation in Japanese and Korean patients with unresectable hepatocellular carcinoma. European journal of cancer. 2011; 47:2117-2127.

34. Lencioni RA, Allgaier HP, Cioni D, Olschewski M, Deibert P, Crocetti L, Frings H, Laubenberger J, Zuber I, Blum HE and Bartolozzi C. Small hepatocellular carcinoma in cirrhosis: randomized comparison of radio-frequency thermal ablation versus percutaneous ethanol injection. Radiology. 2003; 228:235-240.

35. Li C, Zhang W, Zhang R, Zhang L, Wu P and Zhang F. Therapeutic effects and prognostic factors in high-intensity focused ultrasound combined with chemoembolisation for larger hepatocellular carcinoma. European journal of cancer. 2010; 46:2513-2521.

36. Lin SM, Lin CJ, Lin CC, Hsu CW and Chen YC. Radiofrequency ablation improves prognosis compared with ethanol injection for hepatocellular carcinoma $<$ or $=4$ cm. Gastroenterology. 2004; 127:1714-1723.
37. Lin SM, Lin CJ, Lin CC, Hsu CW and Chen YC. Randomised controlled trial comparing percutaneous radiofrequency thermal ablation, percutaneous ethanol injection, and percutaneous acetic acid injection to treat hepatocellular carcinoma of $3 \mathrm{~cm}$ or less. Gut. 2005; 54:1151-1156.

38. Malagari K, Pomoni M, Kelekis A, Pomoni A, Dourakis S, Spyridopoulos T, Moschouris H, Emmanouil E, Rizos $\mathrm{S}$ and Kelekis D. Prospective randomized comparison of chemoembolization with doxorubicin-eluting beads and bland embolization with BeadBlock for hepatocellular carcinoma. Cardiovascular and interventional radiology. 2010; 33:541-551.

39. Moreno-Luna LE, Yang JD, Sanchez W, Paz-Fumagalli R, Harnois DM, Mettler TA, Gansen DN, De Groen PC, Lazaridis KN, Narayanan Menon KV, Larusso NF, Alberts SR, Gores GJ, et al. Efficacy and safety of transarterial radioembolization versus chemoembolization in patients with hepatocellular carcinoma. Cardiovascular and interventional radiology. 2013; 36:714-723.

40. Morimoto M, Numata $\mathrm{K}$, Kondou M, Nozaki A, Morita S and Tanaka K. Midterm outcomes in patients with intermediate-sized hepatocellular carcinoma: a randomized controlled trial for determining the efficacy of radiofrequency ablation combined with transcatheter arterial chemoembolization. Cancer. 2010; 116:5452-5460.

41. Muhammad A, Dhamija M, Vidyarthi G, Amodeo D, Boyd W, Miladinovic B and Kumar A. Comparative effectiveness of traditional chemoembolization with or without sorafenib for hepatocellular carcinoma. World journal of hepatology. 2013; 5:364-371.

42. Nicolini A, Martinetti L, Crespi S, Maggioni M and Sangiovanni A. Transarterial chemoembolization with epirubicin-eluting beads versus transarterial embolization before liver transplantation for hepatocellular carcinoma. Journal of vascular and interventional radiology. 2010; 21:327-332.

43. Peng ZW, Guo RP, Zhang YJ, Lin XJ, Chen MS and Lau WY. Hepatic resection versus transcatheter arterial chemoembolization for the treatment of hepatocellular carcinoma with portal vein tumor thrombus. Cancer. 2012; 118:4725-4736.

44. Peng ZW, Zhang YJ, Chen MS, Xu L, Liang HH, Lin XJ, Guo RP, Zhang YQ and Lau WY. Radiofrequency ablation with or without transcatheter arterial chemoembolization in the treatment of hepatocellular carcinoma: a prospective randomized trial. Journal of clinical oncology. 2013; 31:426-432.

45. Qu XD, Chen CS, Wang JH, Yan ZP, Chen JM, Gong GQ, Liu QX, Luo JJ, Liu LX, Liu R and Qian S. The efficacy of TACE combined sorafenib in advanced stages hepatocellullar carcinoma. BMC cancer. 2012; 12:263.

46. Recchia F, Passalacqua G, Filauri P, Doddi M, Boscarato P, Candeloro G, Necozione S, Desideri G and Rea S. Chemoembolization of unresectable hepatocellular carcinoma: Decreased toxicity with slow-release 
doxorubicin-eluting beads compared with lipiodol. Oncol Rep. 2012; 27:1377-1383.

47. Sacco R, Bargellini I, Bertini M, Bozzi E, Romano A, Petruzzi P, Tumino E, Ginanni B, Federici G, Cioni R, Metrangolo S, Bertoni M, Bresci G, et al. Conventional versus doxorubicin-eluting bead transarterial chemoembolization for hepatocellular carcinoma. Journal of Vascular and Interventional Radiology. 2011; 22:1545-1552.

48. Salem R, Lewandowski RJ, Kulik L, Wang E, Riaz A, Ryu RK, Sato KT, Gupta R, Nikolaidis P, Miller FH, Yaghmai V, Ibrahim SM, Senthilnathan S, et al. Radioembolization results in longer time-to-progression and reduced toxicity compared with chemoembolization in patients with hepatocellular carcinoma. Gastroenterology. 2011; 140:497-507 e492.

49. Shibata T, Isoda H, Hirokawa Y, Arizono S, Shimada $\mathrm{K}$ and Togashi K. Small hepatocellular carcinoma: Is radiofrequency ablation combined with transcatheter arterial chemoembolization more effective than radiofrequency ablation alone for treatment? Radiology. 2009; 252:905-913.

50. Shiina S, Teratani T, Obi S, Sato S, Tateishi R, Fujishima T, Ishikawa T, Koike Y, Yoshida H, Kawabe T and Omata M. A randomized controlled trial of radiofrequency ablation with ethanol injection for small hepatocellular carcinoma. Gastroenterology. 2005; 129:122-130.

51. Shim SJ, Seong J, Han KH, Chon CY, Suh CO and Lee JT. Local radiotherapy as a complement to incomplete transcatheter arterial chemoembolization in locally advanced hepatocellular carcinoma. Liver international. 2005; 25:1189-1196.

52. Song MJ, Chun HJ, Song do S, Kim HY, Yoo SH, Park $\mathrm{CH}$, Bae SH, Choi JY, Chang UI, Yang JM, Lee HG and Yoon SK. Comparative study between doxorubicin-eluting beads and conventional transarterial chemoembolization for treatment of hepatocellular carcinoma. Journal of hepatology. 2012; 57:1244-1250.

53. Song MJ, Park CH, Kim JD, Kim HY, Bae SH, Choi JY, Yoon SK, Chun HJ, Choi BG and Lee HG. (2011). Drugeluting bead loaded with doxorubicin versus conventional Lipiodol-based transarterial chemoembolization in the treatment of hepatocellular carcinoma: a case-control study of Asian patients. Eur J Gastroenterol Hepatol, pp. 521-527.

54. Tan WF, Qiu ZQ, Yu Y, Ran RZ, Yi B, Lau WY, Liu C, Qiu YH, Feng FL, Wang JH, Yan PN, Zhang BH, Wu MC, et al. Sorafenib extends the survival time of patients with multiple recurrences of hepatocellular carcinoma after liver transplantation. Acta pharmacologica Sinica. 2010; 31:1643-1648.

55. Wiggermann P, Sieron D, Brosche C, Brauer T, Scheer F, Platzek I, Wawrzynek W and Stroszczynski C. Transarterial Chemoembolization of Child-A hepatocellular carcinoma: drug-eluting bead TACE (DEB TACE) vs. TACE with cisplatin/lipiodol (cTACE). Medical science monitor. 2011; 17:CR189-195.

56. Wu F, Wang ZB, Chen WZ, Zou JZ, Bai J, Zhu H, Li $\mathrm{KQ}$, Jin $\mathrm{CB}$, Xie FL and Su HB. Advanced hepatocellular carcinoma: treatment with high-intensity focused ultrasound ablation combined with transcatheter arterial embolization. Radiology. 2005; 235:659-667.

57. Yang W, Chen MH, Wang MQ, Cui M, Gao W, Wu W, Wu JY, Dai Y and Yan K. Combination therapy of radiofrequency ablation and transarterial chemoembolization in recurrent hepatocellular carcinoma after hepatectomy compared with single treatment. Hepatology research. 2009; 39:231-240.

58. Zeng ZC, Tang ZY, Fan J, Zhou J, Qin LX, Ye SL, Sun HC, Wang BL, Yu Y, Wang JH and Guo W. A comparison of chemoembolization combination with and without radiotherapy for unresectable hepatocellular carcinoma. Cancer J. 2004; 10:307-316.

59. Minagawa M and Makuuchi M. Treatment of hepatocellular carcinoma accompanied by portal vein tumor thrombus. World journal of gastroenterology. 2006; 12:7561-7567.

60. Yamada R, Sato M, Kawabata M, Nakatsuka H, Nakamura K and Takashima S. Hepatic artery embolization in 120 patients with unresectable hepatoma. Radiology. 1983; 148:397-401.

61. ter Haar G. High intensity ultrasound. Seminars in laparoscopic surgery. 2001; 8:77-89.

62. Iezzi R, Pompili M, Posa A, Coppola G, Gasbarrini A and Bonomo L. Combined locoregional treatment of patients with hepatocellular carcinoma: State of the art. World journal of gastroenterology. 2016; 22:1935-1942.

63. Choe WH, Kim YJ, Park HS, Park SW, Kim JH and Kwon SY. Short-term interval combined chemoembolization and radiofrequency ablation for hepatocellular carcinoma. World journal of gastroenterology. 2014; 20:12588-12594.

64. Kim SW, Oh D, Park HC, Lim do H, Shin SW, Cho SK, Gwak GY, Choi MS, Paik YH and Paik SW. Transcatheter arterial chemoembolization and radiation therapy for treatment-naive patients with locally advanced hepatocellular carcinoma. Radiation oncology journal. 2014; 32:14-22.

65. Yoon SM, Lim YS, Won HJ, Kim JH, Kim KM, Lee HC, Chung YH, Lee YS, Lee SG, Park JH and Suh DJ. Radiotherapy plus transarterial chemoembolization for hepatocellular carcinoma invading the portal vein: longterm patient outcomes. International journal of radiation oncology, biology, physics. 2012; 82:2004-2011.

66. Li XL, Guo WX, Hong XD, Yang L, Wang K, Shi J, Li $\mathrm{N}$, Wu MC and Cheng SQ. Efficacy of the treatment of transarterial chemoembolization combined with radiotherapy for hepatocellular carcinoma with portal vein tumor thrombus: A propensity score analysis. Hepatology research. 2016.

67. Thandassery RB, Goenka U and Goenka MK. Role of local ablative therapy for hepatocellular carcinoma. Journal of clinical and experimental hepatology. 2014; 4:S104-111.

68. Ohnishi K, Nomura F, Ito S and Fujiwara K. Prognosis of small hepatocellular carcinoma (less than $3 \mathrm{~cm}$ ) after percutaneous acetic acid injection: study of 91 cases. Hepatology. 1996; 23:994-1002. 